\title{
ITC Commentary on Metformin Clinical Drug-Drug Interaction Study Design That Enables an Efficacy- and Safety-Based Dose Adjustment Decision
} DOI:

10.1002/cpt.1082

\section{Document Version}

Accepted author manuscript

Link to publication record in Manchester Research Explorer

Citation for published version (APA):

Zamek-Gliszczynski, M. J., Chu, X., Cook, J. A., Custodio, J. M., Galetin, A., Giacomini, K. M., Lee, C. A., Paine, M. F., Ray, A. S., Ware, J. A., Wittwer, M. B., \& Zhang, L. (2018). ITC Commentary on Metformin Clinical DrugDrug Interaction Study Design That Enables an Efficacy-and Safety-Based Dose Adjustment Decision. Clinical Pharmacology and Therapeutics. https://doi.org/10.1002/cpt.1082

\section{Published in:}

Clinical Pharmacology and Therapeutics

\section{Citing this paper}

Please note that where the full-text provided on Manchester Research Explorer is the Author Accepted Manuscript or Proof version this may differ from the final Published version. If citing, it is advised that you check and use the publisher's definitive version.

\section{General rights}

Copyright and moral rights for the publications made accessible in the Research Explorer are retained by the authors and/or other copyright owners and it is a condition of accessing publications that users recognise and abide by the legal requirements associated with these rights.

\section{Takedown policy}

If you believe that this document breaches copyright please refer to the University of Manchester's Takedown Procedures [http://man.ac.uk/04Y6Bo] or contact uml.scholarlycommunications@manchester.ac.uk providing relevant details, so we can investigate your claim.

\section{OPEN ACCESS}




\title{
ITC commentary on metformin clinical drug-drug interaction study design that enables an efficacy- and safety-based dose adjustment decision
}

\author{
Maciej J. Zamek-Gliszczynski* ${ }^{1}$, Xiaoyan Chu $^{2}$, Jack A. Cook ${ }^{3}$, Joseph M. Custodio ${ }^{4}$, \\ Aleksandra Galetin ${ }^{5}$, Kathleen M. Giacomini ${ }^{6}$, Caroline A. Lee ${ }^{7}$, Mary F. Paine ${ }^{8}$, Adrian S. Ray ${ }^{9}$, \\ Joseph A. Ware ${ }^{10}$, Matthias B. Wittwer ${ }^{11}$, and Lei Zhang ${ }^{12}$ \\ on behalf of the International Transporter Consortium
}

1. Quantitative Drug Disposition, GlaxoSmithKline plc, 709 Swedeland Road, King of Prussia, PA, 19406, USA; maciej.x.zamek-gliszczynski@ gsk.com; 610-270-6278

2. Department of Pharmacokinetics, Pharmacodynamics and Drug Metabolism, Merck \& CO., Inc, Kenilworth, NJ 07033, USA; xiaoyan_chu@merck.com 732-594-0977

3. Clinical Pharmacology, Global Product Development, Pfizer Inc., Groton, CT, USA; jack.cook@pfizer.com

4. Clinical Pharmacology, Gilead Sciences, Inc., 333 Lakeside Drive, Foster City, CA, 94404, USA; joseph.custodio@gilead.com; 650-372-7065

5. Centre for Applied Pharmacokinetic Research, School of Health Sciences, The University of Manchester, Stopford Building, Oxford Road, Manchester M13 9PT, UK;

aleksandra.galetin@manchester.ac.uk; +44-161-275-6886

6. Department of Bioengineering and Therapeutic Sciences, Schools of Pharmacy and Medicine, University of California, San Francisco, CA, USA; kathy.giacomini @ ucsf.edu

7. Drug Metabolism and Clinical Pharmacology, DMPK Solutions, San Diego, CA, USA; dmpksolutions.clee@gmail.com; 858-449-1797

8. Department of Pharmaceutical Sciences, College of Pharmacy, Washington State University, Spokane, WA 99210, USA; mary.paine@ wsu.edu; 509-358-7759

9. Clinical Research, Gilead Sciences, Inc., 333 Lakeside Drive, Foster City, CA, 94404, USA; adrian.ray@gilead.com; 650-200-6770

10. Clinical Pharmacology, Genentech, 1-DNA Way, MS-463A, South San Francisco, CA 94080, USA; ware.joseph@gene.com;650-467-3123

11. Roche Pharma Research and Early Development, Pharmaceutical Sciences, Roche Innovation Center Basel, F. Hoffmann-La Roche Ltd, Grenzacherstrasse 124, CH-4070 Basel, Switzerland; matthias.wittwer@ roche.com

12. Office of Research and Standards, Office of Generic Drugs, Center for Drug Evaluation and Research, Food and Drug Administration, 10903 New Hampshire Ave, Silver Spring, MD 20993, USA; leik.zhang@fda.hhs.gov; 301-796-1635 
*Corresponding Author:

Maciej J. Zamek-Gliszczynski, Ph.D.

709 Swedeland Road

Office 27.3056, Mailstop UW2732

King of Prussia, PA, 19406, USA

Tel: 610-270-6278

E-mail: maciej.x.zamek-gliszczynski@gsk.com

Format: Commentary

Text: 1,661/1,600 words

Introduction: $75 / 75$ words

References: $10 / 10$

Figures/Tables: 2/2

Conflict of Interest: As an Associate Editor for Clinical Pharmacology \& Therapeutics, Mary Paine was not involved in the review or decision process for this paper.

Funding: Dr. Giacomini's research on metformin is supported in part by a grant from the National Institute of General Medical Sciences, GM117163.

Disclaimer: The contents of this manuscript reflect the views of the authors and should not be construed to represent the FDA's views or policies. No official support or endorsement by the FDA is intended or should be inferred. The mention of commercial products, their sources, or their use in connection with material reported herein is not to be construed as either an actual or implied endorsement of such products by the FDA.

Abbreviations: drug-drug interaction (DDI), multidrug and toxin extrusion protein (MATE), new molecular entity (NME), organic cation transporter (OCT), oral glucose tolerance test (OGTT), area under the concentration-time curve (AUC) 


\section{Introduction $(75 / 75$ words $)$}

Metformin DDI studies are conducted during development of drugs that inhibit OCTs/MATEs. Monitoring solely changes in systemic exposure, the typical DDI study endpoint, appears inadequate for metformin, which is metabolically stable, has poor passive membrane permeability, and undergoes transporter-mediated tissue distribution and clearance. Evaluation of renal clearance, antihyperglycemic effects, and potentially lactate as exploratory safety marker, can support rational metformin dose adjustment. The proposed DDI study design aims to adequately inform metformin dosing during co-medication. 
Metformin is a hydrophilic, metabolically-stable drug with minimal passive membrane permeability, resulting in transporter-mediated distribution and clearance (Figure 1 [1]). Hepatic sinusoidal uptake by organic cation transporter (OCT) 1 is a key determinant of metformin distribution to the liver, the primary site of anti-hyperglycemic effect and rare serious toxicity, lactic acidosis (1). However, metformin is not cleared by the liver; it is cleared primarily by renal active tubular secretion via OCT2/MATE1/MATE2-K (1). Transporter-mediated disposition of metformin makes it susceptible to mechanistically atypical DDIs, with the potential to independently affect metformin pharmacokinetics, efficacy, or safety when coadministered with drugs that inhibit these transporters (2-6).

Metformin renal DDI studies have been conducted routinely during development of drugs that inhibit OCT2/MATE1/2-K since 2010-2012 per revision of draft guidances issued by regulatory authorities, including the EMA and FDA. The endpoint suggested in these guidances, change in systemic drug exposure, has been most commonly studied to-date for metformin DDI studies. This sole endpoint is uniquely inappropriate for evaluating DDI effect on metformin because of its transporter-mediated distribution and clearance (1). Accordingly, dose-adjustment decisions for metformin based solely on systemic exposure changes may not be appropriate, because systemic exposure may not represent the DDI effect at the site of action (2-6). That is, metformin pharmacodynamics may change independently of systemic pharmacokinetics, because distribution to the hepatic site of action is mediated by OCT1, which can be subject to independent modulation during DDIs. Metformin DDIs can be mechanistically complex, as changes in metformin pharmacodynamics can be unexpected based on observed (or lack of) changes in systemic pharmacokinetics. Specifically, altered hepatic exposure and antihyperglycemic effect despite unaltered systemic pharmacokinetics, and vice versa (i.e., unaltered 
hepatic exposure and response during increased systemic exposure), have been reported (2-6). This commentary proposes a metformin clinical DDI study design that enables a rational dose adjustment decision for metformin when co-administered with OCT/MATE inhibitors.

\section{Metformin DDI Study Design}

Compared with most pharmacokinetic DDI studies, metformin DDI studies are unique in that both pharmacokinetic and pharmacologic endpoints are needed to enable rational dose adjustment. Such studies should examine three metformin endpoints: 1) systemic pharmacokinetics, 2) renal clearance, and 3) anti-hyperglycemic effects [i.e., oral glucose tolerance test (OGTT)]. Metformin effect on blood lactate concentrations may also be evaluated to assess potential for lactic acidosis, although this approach is not clinically validated.

The OGTT is administered as a 75-g glucose dose 1-3 hours post metformin administration. This effect endpoint is reported as reduction of glucose area under the concentration-time curve (AUC), which requires a crossover glucose AUC determination following an OGTT administered in the absence of metformin. This design enables examination of metformin anti-hyperglycemic effect acutely in healthy volunteers. The OGTT is a favored marker of metformin anti-hyperglycemic effect because it is easily administered, and effects can be observed after a single metformin dose. Finally, this approach evaluates potential alterations in overall anti-hyperglycemic effect, not just at the level of the liver (largest contributor to metformin response), but also in the intestine and skeletal muscle, although the latter should not be mechanistically attributed to OCT1/2 and MATE1/2-K modulation, because they involve additional transporters (1). Other endpoints have shortcomings. First, because metformin does not elicit hypoglycemia in healthy volunteers, fasting blood glucose is not appropriate for these 
individuals. Second, although metformin effect could be measured as reductions in fasting blood glucose or hemoglobin A1c in diabetic patients, neither are practical in the context of a DDI study due to large patient (vs. healthy volunteers) numbers required for robust measurement of a variable biomarker (fasting blood glucose) or long study durations necessary to detect changes in hemoglobin A1c. Finally, because metformin is not an insulin secretagogue, insulin is not an appropriate biomarker.

Blood lactate is most useful as an exploratory safety marker when studied with the highest metformin clinical dosing regimen (ideally $850 \mathrm{mg}$ TID, although $1000 \mathrm{mg}$ BID may be more practical to enable sampling over a 12-hour vs. 8-hour dosing interval). The recommendation to use the highest clinical dose of metformin is unusual in the context of a DDI study, where generally the lowest dose is used to ensure safety when systemic exposure is increased. However, this approach is considered safe with study period durations $\leq 1$ week, and for metformin, the most aggressive dosing regimen is needed to determine whether ultimately a dose limitation may be warranted because of increased likelihood of lactic acidosis. However, blood lactate is difficult to measure, has not yet been clinically validated, and may not show a response in this context. Furthermore, lactate response may be correlated with antihyperglycemic effect.

Evaluation of metformin DDIs requires a unique 3-way crossover design (Figure 2). The three study periods are 1) NME alone, 2) metformin alone, and 3) metformin with NME. The first period is necessary to characterize any independent effects of the NME on antihyperglycemic response. If this endpoint is impacted during co-administration (third period), these data enable understanding whether observed changes are due to altered metformin disposition, dual response, or a combination of both. If NME anti-hyperglycemic effects can be 
ruled out with existing blood glucose and/or hemoglobin A1c, a traditional two-period study may suffice.

The DDI should be evaluated with steady-state metformin alone and with the NME at clinically-relevant steady-state concentrations (typically one week of dosing per period, unless the NME has a long half-life, requiring dosing for a minimum of five half-lives). Alternatively, metformin DDIs can be evaluated with acute metformin [Figure 2b (4)], although it is not known presently whether acute metformin DDI results can be extrapolated to the steady-state situation, or whether acute metformin dosing is sufficient for assessment of lactate response. Metformin is available in immediate- and extended-release formulations; immediate release is recommended, as it is the most commonly used.

The number of subjects needed to describe a metformin DDI with adequate precision must consider the two pharmacokinetic endpoints (systemic exposure and renal clearance) and two effect endpoints (glucose and potentially lactate), which is uniquely complex relative to routine DDI study designs. For metformin pharmacokinetic parameters, a study to conclude noeffect should target the standard bioequivalence boundaries for systemic exposure and renal clearance ratios $(0.8-1.25)$, with specific subject numbers driven by metformin pharmacokinetic variability. In terms of anti-hyperglycemic effect, metformin dose is titrated in 500-mg increments and a typical therapeutic dose is approximately $1500 \mathrm{mg}$. As such, a threshold change in anti-hyperglycemic effect is defined as one requiring a $500 \mathrm{mg}$ metformin dose adjustment, with preliminary conservative OGTT no-effect boundaries of 0.75-1.33. Current understanding of lactate is insufficient to define no-effect boundaries. For drugs likely to alter either the PK or PD more than the proposed no-effect criteria, the precision of the study should be sufficient to justify subsequent dosing recommendations. 
Modeling and Simulation in Metformin DDI Study Design

A physiologically-based pharmacokinetic model for metformin has been proposed and verified to a limited extent (7). Confidence in this approach is limited due to complex interplay of multiple transporters and difficulties in validating model-predicted changes in tissue exposure. However, the availability of well-designed metformin clinical DDI data that include effect measurements, as well as emerging metformin tissue pharmacokinetic data from clinical imaging studies (6), should allow refinement and verification of these models to achieve a qualified model that could be used to prospectively guide metformin DDI studies in the future.

\section{Pharmacogenetic Considerations}

Transporters that mediate metformin renal clearance (OCT2, MATE1, and MATE2-K) and hepatic distribution (OCT1 and MATE1) exhibit a relatively high prevalence of functional polymorphisms, including functionally-deficient phenotypes $(6,8)$. For example, up to $9 \%$ of Caucasians exhibit an OCT1 null phenotype (6). Pharmacogenetic variability in metformin pathways raises an important question whether and how it should be considered in DDI study design. Based on currently available information, it is premature to make specific pharmacogenetic recommendations, as there is no evidence suggesting that incorporating pharmacogenetic recruitment criteria would be helpful in evaluating metformin DDIs. Instead, crossover design studies are emphasized. Nevertheless, genotyping study subjects would be prudent to help explain outlier individuals, and over time, help support a potential value (or lack thereof) for genotype-based metformin DDI study design, as well as improve the understanding 
of the impact of individual and combined covariates causing changes in metformin pharmacokinetics and pharmacodynamics.

\section{Value of Proposed Design to Inform Metformin Dosing}

The following case study illustrates the value of the proposed metformin DDI study design in dose adjustment recommendations. An OCT2-inhibitor NME increased metformin systemic exposure 2.45-fold; no other endpoints were measured (9). The U.S. product labeling recommended limiting co-administered metformin to the lowest clinical dose out of concerns for increased lactic acidosis potential. The rationale for this recommendation was that the DDI study supported metformin safety only with $500 \mathrm{mg}$ BID, where metformin systemic exposure during co-administration approximated the highest approved dose regimen (850 mg TID). Furthermore, the pharmacologic relevance of the observed DDI was impossible to explain in the prescribing information based on the change in systemic exposure alone. Therefore, it was recommended to monitor metformin effect when starting and discontinuing the co-medication. Finally, the pharmacokinetics of this DDI are puzzling, because metformin half-life was not increased as would be expected for an inhibitor of renal clearance via OCT2; however, as this parameter was not obtained, it is unknown whether renal clearance was decreased as expected and was the sole reason for the increased systemic exposure. If the DDI study additionally assessed renal clearance and metformin effect as proposed, a better understanding of the DDI and its consequences may have been obtained to support a dosing recommendation.

Another recent metformin DDI study illustrates the value of the proposed design in characterizing a complex metformin DDI, which would have been overlooked in a traditional protocol due to unchanged metformin systemic exposure (4). Famotidine enhanced the anti- 
hyperglycemic effects of metformin in the first 30 minutes after administration. The study evaluated the independent anti-hyperglycemic effects of famotidine to rule out a potential pharmacodynamic interaction. Famotidine increased both metformin oral absorption and renal clearance to the same extent, such that systemic exposure was unaltered, but elucidation of these pharmacokinetic changes was possible only because urinary metformin recovery and renal clearance were studied. A similarly complex interaction including increased metformin antihyperglycemic effect was observed with the MATE2-K inhibitor nizatidine; increased metformin half-life was attributed to increased volume of distribution rather than reduced renal clearance (10).

\section{Concluding Remarks}

Due to its extremely hydrophilic nature and transporter-mediated tissue distribution/clearance, metformin requires a non-traditional DDI study protocol that examines its effect (OGTT) and renal clearance in addition to systemic pharmacokinetics. The proposed study protocol is specific to the labeling of OCT/MATE inhibitor NMEs for co-administration with metformin. Results from these studies should not be extrapolated to other drugs. Conversely, while other probe drugs or biomarkers (e.g., creatinine) may provide a qualitative assessment of the potential for an effect on the renal clearance of metformin, they are unlikely to be informative for the distribution to the liver and resulting efficacy and safety of metformin. 


\section{Figures}

Figure 1. Scheme depicting OCT/MATE transporters governing metformin hepatic distribution and renal clearance. Note that the liver is the primary site of metformin's anti-hyperglycemic effect and its most serious toxicity, lactic acidosis, but it is not a clearing organ for metformin; the kidney is the major clearing organ. OCT, organic cation transporter; MATE, multidrug and toxin extrusion protein.

Figure 2. Generic scheme of the proposed metformin DDI study design (A). The depicted study sequence is arbitrary, and ideally an equal number of subjects would be randomized into possible study sequence combinations. Alternatively, metformin DDIs can be evaluated with acute metformin administration (B). Recent famotidine DDI study used this acute metformin design DDI study (4), where a single $1000 \mathrm{mg}$ metformin dose was followed by an 850mg dose 12 hours later. The presented design assumes QD dosing of NME for one week, but this can be abbreviated if clinically-relevant steady state can be achieved faster, as exemplified in the famotidine DDI study (4). Note that it is not known whether acute metformin DDI results can be extrapolated to the steady-state situation, or whether acute metformin dosing is sufficient for assessment of lactate response. NME, new molecular entity; OGTT, oral glucose tolerance test; PK, pharmacokinetics; CL,r, renal clearance. Black arrows denote metformin administration; green arrows exemplify NME administration intended for QD dosing. 


\section{References}

(1) Gong, L., Goswami, S., Giacomini, K.M., Altman, R.B. \& Klein, T.E. Metformin pathways: pharmacokinetics and pharmacodynamics. Pharmacogenet Genomics 22, 8207 (2012).

(2) Cho, S.K., Kim, C.O., Park, E.S. \& Chung, J.Y. Verapamil decreases the glucoselowering effect of metformin in healthy volunteers. Br J Clin Pharmacol 78, 1426-32 (2014).

(3) Cho, S.K. et al. Rifampin enhances the glucose-lowering effect of metformin and increases OCT1 mRNA levels in healthy participants. Clin Pharmacol Ther 89, 416-21 (2011).

(4) Hibma, J.E. et al. The Effect of Famotidine, a MATE1-Selective Inhibitor, on the Pharmacokinetics and Pharmacodynamics of Metformin. Clin Pharmacokinet 55, 711-21 (2016).

(5) Higgins, J.W., Bedwell, D.W. \& Zamek-Gliszczynski, M.J. Ablation of both organic cation transporter (OCT)1 and OCT2 alters metformin pharmacokinetics but has no effect on tissue drug exposure and pharmacodynamics. Drug Metab Dispos 40, 1170-7 (2012).

(6) Sundelin, E. et al. Genetic Polymorphisms in Organic Cation Transporter 1 Attenuates Hepatic Metformin Exposure in Humans. Clin Pharmacol Ther 102, 841-8 (2017).

(7) Burt, H.J. et al. Metformin and cimetidine: Physiologically based pharmacokinetic modelling to investigate transporter mediated drug-drug interactions. Eur J Pharm Sci 88, 70-82 (2016).

(8) Stocker, S.L. et al. The effect of novel promoter variants in MATE1 and MATE2 on the pharmacokinetics and pharmacodynamics of metformin. Clin Pharmacol Ther 93, 18694 (2013).

(9) Song, I.H. et al. The Effect of Dolutegravir on the Pharmacokinetics of Metformin in Healthy Subjects. J Acquir Immune Defic Syndr 72, 400-7 (2016).

(10) Morrissey, K.M., Stocker, S.L., Chen, E.C., Castro, R.A., Brett, C.M. \& Giacomini, K.M. The Effect of Nizatidine, a MATE2K Selective Inhibitor, on the Pharmacokinetics and Pharmacodynamics of Metformin in Healthy Volunteers. Clin Pharmacokinet 55, 495506 (2016). 


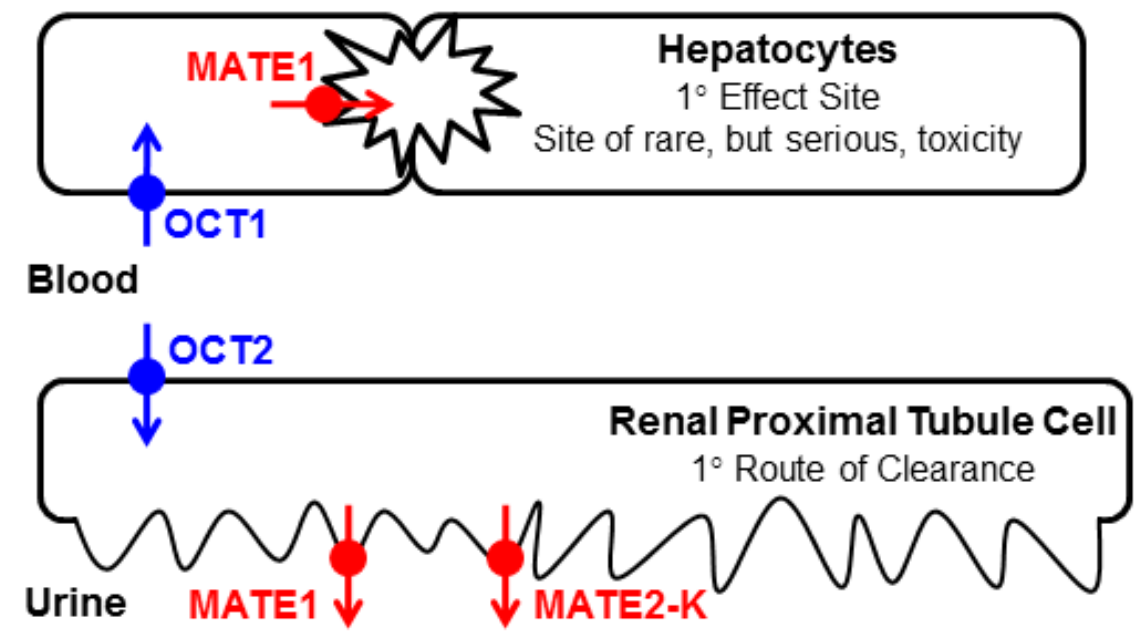


